\title{
Tratamiento térmico alternativo para el metal de soldadura de un acero $9 \mathrm{Cr}$
}

Ariel Burgos ${ }^{1}$, Johnnatan Rodríguez ${ }^{2}$, Hernán Svoboda $^{3}$, Estela Surian ${ }^{4}$

\footnotetext{
${ }^{1}$ Universidad Nacional de Lomas de Zamora, Facultad de Ingeniería, Camino de Cintura y Juan XXIII, (1832) Lomas de Zamora, Buenos Aires, Argentina. e-mail: arielburgo@gmail.com

${ }^{2}$ Laboratorio de Caracterización y Procesamiento de Metales (CPM), Laboratorio Nacional de Nanotecnología (LNnano), Centro Nacional de Investigación en Energía y Materiales (CNPEM), 13083-970, Campinas, São Paulo, SP, Brasil.

${ }^{3}$ GTSyCM$^{3}$, INTECIN, Facultad de Ingeniería - Universidad de Buenos Aires - CONICET, Av. Las Heras 2214 (1427) ciudad Autónoma de Buenos Aires (CABA), Argentina - Av. Rivadavia 1917, Ciudad Autónoma de Buenos Aires (CABA), Buenos Aires, Argentina.

${ }^{4}$ Investigadora y consultora independiente, Ciudad Autónoma de Buenos Aires (CABA), Buenos Aires, Argentina.
}

\section{RESUMEN}

Los aceros 9Cr con resistencia al creep mejorada (CSE9Cr) son ampliamente utilizados en la industria de generación termoeléctrica, en los que la soldabilidad es un factor crítico, debido a que se los emplea en la fabricación de equipos de construcción soldada. Para mejorar la resistencia al creep se busca obtener microestructuras que contengan alta densidad de dislocaciones y finos precipitados que refuercen la matriz martensítica a alta temperatura. Estas microestructuras pueden ser alcanzadas luego de aplicar un tratamiento térmico de post soldadura (PWHT), por cuanto su optimización es importante. En este trabajo se estudió la aplicación de un PWHT alternativo a los industrialmente utilizados sobre depósitos de soldadura de aceros CSE9Cr. Se soldaron cupones de metal de aporte puro (MAP) con alambres tubulares flux-cored experimentales. Los PWHT evaluados fueron: 1) convencional: revenido a $760^{\circ} \mathrm{C}$ durante 2 horas, 2) alternativo: solubilizado a $1100^{\circ} \mathrm{C}$ durante 1 hora + primer revenido a $660^{\circ} \mathrm{C}$ durante 3 horas + segundo revenido a $660^{\circ} \mathrm{C}$ durante 3 horas. Sobres muestras tratadas, se llevaron a cabo ensayos de tracción en caliente (HTT) a diferentes velocidades de deformación inicial y temperaturas, analizando las microestructuras de partida por microscopía SEM y EBSD. Se extrapolaron los resultados de HTT aplicando la teoría de correlación HTT-Creep y de parametrización Larson-Miller. Se encontró que un PWHT de solubilizado más un doble revenido mejoró las propiedades a elevada temperatura. Esto estaría asociado a la obtención de una microestructura con una mayor densidad de dislocaciones y precipitados finos que limitaron el movimiento de las dislocaciones en el tiempo.

Palabras clave: aceros $9 \mathrm{Cr}$ con resistencia al creep mejorada, FCAW, PWHT, microestructura, propiedades mecánicas a alta temperatura.

\section{ABSTRACT}

The creep strength enhanced $9 \mathrm{Cr}$ steels (CSE9Cr) are extremely important in the thermoelectric generation industry. The weldability of these alloys is critical as they are used in welded constructed equipments. A microstructure containing a high dislocation density and fine precipitates that reinforce the martensitic matrix is required to improve their creep strength. Mechanical properties are achieved after post weld heat treatments (PWHT), then the optimization of this PWHT is important. An alternative PWHT on deposits of CSE9Cr was studied. All weld metal coupons (AWM) were welded with experimental flux-cored tubular wires. The PWHT's analyzed were: 1) conventional tempering $\left.\left(760^{\circ} \mathrm{C} \times 2 \mathrm{~h}\right), 2\right)$ alternative: solubilizing $\left(1100{ }^{\circ} \mathrm{C} \times 1 \mathrm{~h}\right)$ + first tempering $\left(660{ }^{\circ} \mathrm{C} \times 3 \mathrm{~h}\right)+$ second tempering $\left(660{ }^{\circ} \mathrm{C} \times 3 \mathrm{~h}\right)$. The obtained microstructures were analyzed by SEM and EBSD. Hot tensile tests (HTT's) at different initial strain rates and temperatures were performed on PHWT samples. HTT's results were extrapolated using the correlation theory HTT-Creep and Larson-Miller parameterization. The results show that mechanical properties were improved due to the alternative PWHT. Possibly a microstructure with a higher density of dislocations and fine precipitates managed to retain the movement of dislocations for longer times.

Keywords: creep strength enhanced 9Cr steel, FCAW, PWHT, microstructure, high temperature mechanical properties. 


\section{INTRODUCCIÓN}

El desafío de las nuevas plantas de generación termoeléctrica es elevar la temperatura y la presión del vapor dentro de su ciclo termodinámico para poder incrementar su rendimiento, reduciendo por lo tanto el costo asociado al combustible y las emisiones de $\mathrm{CO}_{2}$ al medioambiente. Para un rango de funcionamiento a temperaturas de $625-650^{\circ} \mathrm{C}$ y presiones de $30-35 \mathrm{MPa}$, se requiere la utilización de aceros resistentes al creep. Variaciones en la composición química de los aceros $9 \mathrm{Cr}$ permitieron elevar su resistencia mediante el agregado de W a expensas de una reducción en el contenido de Mo y adiciones de B [1] logrando de esta forma disminuir la degradación de la microestructura, reflejada en una disminución de la velocidad mínima de creep y un aumento del tiempo a la rotura [2-4]. Dado que los equipos donde se emplean estos materiales son de construcción soldada, la soldabilidad de estos materiales es relevante y el desarrollo del metal de aporte para su unión presenta los mismos desafíos que el metal base. La microestructura, la composición química, la historia térmica y los mecanismos de endurecimiento actuantes son los factores que controlan las propiedades mecánicas de las soldaduras de aceros 9Cr con resistencia al creep mejorada (CSE9Cr). Una microestructura de pre-servicio martensítica conteniendo obstáculos que retarden el movimiento de las dislocaciones tales como bordes de sub-granos, dislocaciones libres dentro de los sub-granos y carburos del tipo $\mathrm{M}_{23} \mathrm{C}_{6} \mathrm{y}$ MX finamente dispersos, sumados a los efectos de endurecimiento por solución sólida del Mo y W en la matriz, contribuyen a mejorar la resistencia al creep de este metal de soldadura [5]. Los mecanismos de endurecimiento actuantes atribuidos a estos obstáculos son:

1. Endurecimiento por dislocaciones: la presencia de sub-estructuras de dislocaciones son un fuerte impedimento al creep del material ya que se necesita de suficiente energía como para poder desarticular la red que éstas conforman. Por sí sola esta red no genera un endurecimiento significativo, se requiere de su combinación con mecanismos de endurecimiento por partículas para lograr una reducción efectiva de la velocidad mínima de creep [5].

2. Endurecimiento por partículas: partículas en segundas fases $\left(\mathrm{M}_{23} \mathrm{C}_{6} \mathrm{y} \mathrm{MX}\right)$ proveen de un buen anclaje a las dislocaciones retardando el inicio de su movimiento. Ellas reducen la velocidad con la que esta sub-estructura de dislocaciones se desarticula reteniendo por más tiempo los mecanismos de endurecimiento por dislocaciones. En condiciones de creep, estas partículas se engrosan y coalescen perdiendo toda su capacidad de retención. De acuerdo a la velocidad con que estas partículas se degraden se tendrá una reducción de la velocidad mínima de creep [6].

3. Endurecimiento por solución sólida: W y Mo en solución sólida con la matriz retardan por sí solos la degradación de la microestructura en ausencia de los mecanismos de endurecimiento por dislocaciones y por partículas [5]. Durante el creep, estos átomos tienden a difundir hacia $\operatorname{los} \mathrm{M}_{23} \mathrm{C}_{6}$ ya que son formadores de carburos, debilitando la matriz y contribuyendo a la degradación de estas microestructuras.

Se requiere un tratamiento térmico de post soldadura (PWHT) en los aceros CSE9Cr para lograr una microestructura con los obstáculos necesarios para que actúen los mecanismos de endurecimiento durante el trabajo a alta temperatura. En general, los PWHT para estos materiales consisten en el revenido de la estructura martensítica a una temperatura por debajo de $\mathrm{A}_{\mathrm{C} 1}$ [7]. Existen estudios donde también se han aplicado tratamientos térmicos alternativos a estos aceros CSE9Cr que involucran un solubilizado. Con ellos, se busca lograr, a través de un aumento de la temperatura de solubilizado y la aplicación de un doble revenido a temperaturas más bajas [7], una mayor cantidad de obstáculos que refuercen los mecanismos de endurecimiento.

La necesidad de obtener datos de resistencia al creep en tiempos cortos, ha provocado la búsqueda de alternativas a este ensayo, siendo el de tracción en caliente (HTT) una buena opción. Basados en la premisa de que las deformaciones plásticas características son las mismas en ambos ensayos (HTT-resistencia al creep), existen trabajos que correlacionan los resultados de ambos siendo éstos una aproximación satisfactoria con cierto grado de precisión [8-12].

Actualmente, son pocos los consumibles que ajustan la composición química de sus depósitos a la de los CSE9Cr. La gran mayoría se encuentran en plena etapa de desarrollo y en la literatura no se reportan trabajos sistemáticos con alambres tubulares experimentales para soldar bajo el proceso de soldadura semiautomática con protección gaseosa (FCAW-G) en donde se apliquen PWHT's alternativos.

En este trabajo se estudió el efecto de un PWHT alternativo, en comparación con uno convencional, sobre la microestructura y las propiedades mecánicas de metal de aporte puro (MAP - depósito de consumible sin dilución con el metal base) de soldadura de un acero CSE9Cr para el servicio a alta temperatura empleando alambres tubulares aplicando el proceso FCAW-G. 


\section{MATERIALES Y MÉTODOS}

\subsection{Soldaduras}

Se soldó un cupón de MAP según norma ANSI/AWS A5.29/A5.29M:2010 [13], depositando cinco capas con una secuencia de 2, 2, 2, 2 y 3 cordones en cada una, con un alambre tubular del tipo "flux cored" (con relleno de fundente) de $1,2 \mathrm{~mm}$ de diámetro y bajo protección gaseosa de $\mathrm{Ar}-20 \% \mathrm{CO}_{2}$, de formulación experimental. El enmantecado sobre el metal base, utilizado para impedir la dilución del metal aportado con el metal base, fue confeccionado con el mismo consumible. Las soldaduras se realizaron con corriente continua en polaridad electrodo positivo (DCEP), en posición plana y con un caudal de gas de $20 \mathrm{~L} / \mathrm{min}$. La longitud libre de alambre fue de 12 a $15 \mathrm{~mm}$. En la Figura 1 se observa el esquema constructivo del cupón de MAP elaborado.

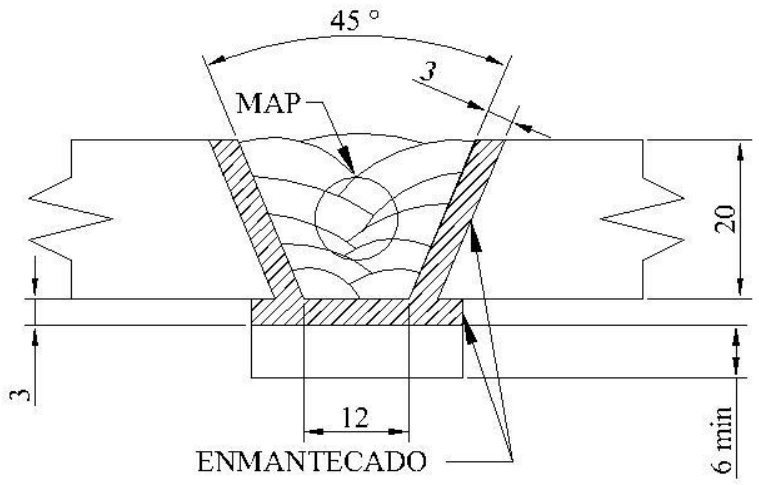

Figura 1: Probeta de MAP según ANSI/AWS A5.29/A5.29M:2010. Dimensiones en mm.

La temperatura de precalentamiento y de entre pasadas fue de $240^{\circ} \mathrm{C}+/-10^{\circ} \mathrm{C}$. La Tabla 1 muestra el promedio de los parámetros de soldadura usados, así como el calor aportado calculado.

Tabla 1: Promedio de los parámetros de soldadura usados.

\begin{tabular}{l|l|l|l|l|l}
\hline $\begin{array}{l}\text { NÚMERO DE } \\
\text { CAPAS }\end{array}$ & $\begin{array}{l}\text { NÚMERO DE } \\
\text { CORDONES }\end{array}$ & $\begin{array}{l}\text { CORRIENTE } \\
\text { [A] }\end{array}$ & $\begin{array}{l}\text { VOLTAJE } \\
{[\mathrm{V}]}\end{array}$ & $\begin{array}{l}\text { VELOCIDAD DE SOLDADURA } \\
{[\mathrm{mm} / \mathbf{s}]}\end{array}$ & $\begin{array}{l}\text { CALOR APORTADO } \\
{[\mathrm{kJ} / \mathrm{mm}]}\end{array}$ \\
\hline 5 & 11 & 218 & 31,0 & 5,6 & 1,2 \\
\hline
\end{tabular}

El MAP fue radiografiado para determinar la calidad de los depósitos.

\subsection{Composición química}

Sobre un corte transversal del MAP se determinó la composición química del depósito por espectrometría de emisión óptica (OES), en la zona de la última capa depositada.

\subsection{Tratamientos Térmicos Post Soldadura}

Sobre muestras de MAP se aplicaron un PWHT de revenido convencional $[14,15]$ y otro alternativo (ver Tabla 2). Este último tuvo por objetivo obtener una microestructura más estable en el creep conteniendo mayor densidad de dislocaciones y una más alta fracción en volumen de precipitados finos del tipo MX. El PWHT alternativo aplicado fue previamente utilizado con éxito por Yin Y. F et al. [7] en aceros similares, en condición de laminado. 
Tabla 2: PWHT aplicado a las muestras de MAP.

\begin{tabular}{l|l|l|l|l|l|l}
\hline \multirow{2}{*}{ PWHT } & \multicolumn{2}{l|}{ SOLUBILIZADO } & \multicolumn{2}{l|}{ REVENIDO } & \multicolumn{2}{l}{ DOBLE REVENIDO } \\
\cline { 2 - 7 } & $\begin{array}{l}\text { Temperatura } \\
{\left[{ }^{\circ} \mathrm{C}\right]}\end{array}$ & $\begin{array}{l}\text { Tiempo } \\
{[\mathrm{h}]}\end{array}$ & $\begin{array}{l}\text { Temperatura } \\
{\left[{ }^{\circ} \mathrm{C}\right]}\end{array}$ & $\begin{array}{l}\text { Tiempo } \\
{[\mathrm{h}]}\end{array}$ & $\begin{array}{l}\text { Temperatura } \\
{\left[{ }^{\circ} \mathrm{C}\right]}\end{array}$ & $\begin{array}{l}\text { Tiempo } \\
{[\mathrm{h}]}\end{array}$ \\
\hline Convencional & - & - & 760 & 2 & - & - \\
\hline Alternativo & 1100 & 1 & 660 & 3 & 660 & 3 \\
\hline
\end{tabular}

\subsection{Microscopia electrónica de barrido (SEM) y análisis de difracción de electrones retrodifundidos (EBSD)}

Cortes transversales de las muestras tratadas térmicamente fueron preparados mediante un proceso tradicional de desbaste y pulido mecánico para examen macroscópico y SEM a fin de analizar la microestructura resultante en MAP.

Otros cortes transversales fueron preparados por electropulido para analizar por EBSD el impacto de los PWHT sobre la estructura cristalina de la fase principal $(\alpha-\mathrm{Fe})$ identificando el tamaño de su dominio cristalino, su misorientación cristalográfica y algunos resultados referentes a bordes de bajo, medio y alto ángulo.

\subsection{Ensayos de tracción en caliente (HTT)}

Se mecanizaron probetas en la dirección transversal al cordón de soldadura según la norma ASTM E 8M-01 [16] escalando su geometría de acuerdo con requerimientos específicos del equipo utilizado (Figura 2). Dichas probetas se ensayaron a 600 y $625^{\circ} \mathrm{C}$ y a velocidades de deformación inicial de $1,39 \mathrm{E}-02 ; 2,78 \mathrm{E}-03$; $2,78 \mathrm{E}-04$ y $5,56 \mathrm{E}-05$ 1/s.

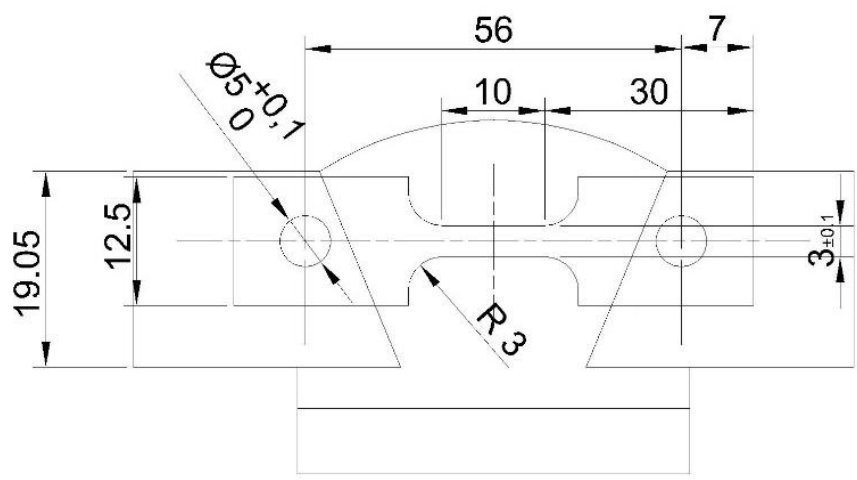

Figura 2: Plano dimensionamiento y esquema de la posición de extracción probeta plana de tracción en caliente (HTT). Dimensiones en mm.

A partir de los resultados obtenidos de los ensayos de HTT y se obtuvieron valores de rotura a creep (CRT) utilizando la teoría de correlación HTT-creep [8-12] y el modelo de Larson-Miller.

\section{RESULTADOS Y DISCUSIÓN}

\subsection{Soldaduras}

En la Figura 3 se puede observar una macrografía de un corte transversal del cupón soldado. De acuerdo con los requerimientos de la norma AWS A5.29/A5.29M:2010 [13]. Se registró un bajo nivel de defectos macroscópicos (poros, inclusiones de escoria, fisuras, etc.) como se pudo corroborar también en la radiografía del cordón completo. 


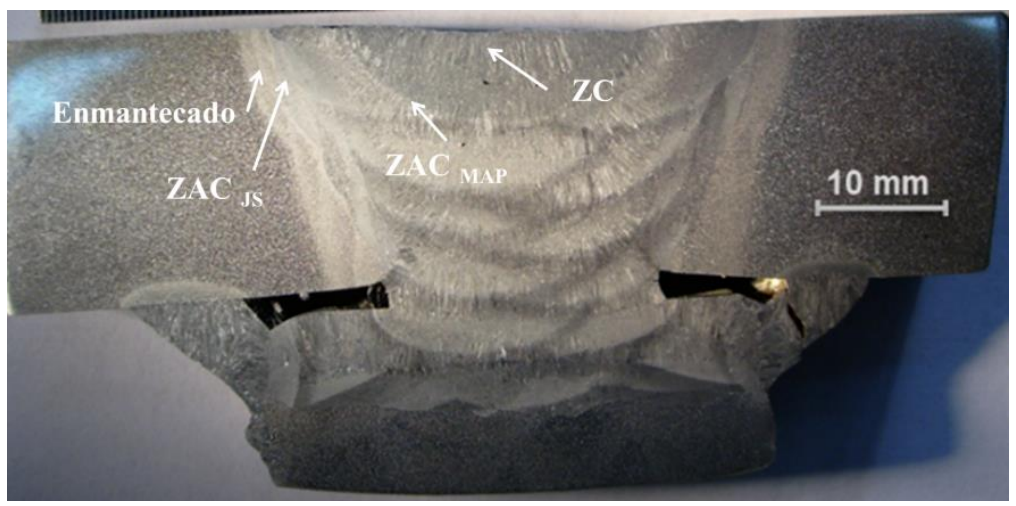

Figura 3: Macrografía del cupón de MAP. ZC - zona columnar; $\mathrm{ZAC}_{\mathrm{MAP}}$ - zona afectada por el calor en la unión de dos cordones dentro del MAP; $\mathrm{ZAC}_{\mathrm{JS}}$ - zona afectada por el calor en la unión con el enmantecado.

\subsection{Composición química del MAP}

La Tabla 3 muestra los resultados del análisis químico obtenido sobre la superficie de la última capa depositada (capa $\left.\mathrm{N}^{\circ} 5\right)$.

Tabla 3: Composición química del MAP (\% en peso).

\begin{tabular}{l|l|l|l|l|l|l|l|l}
\hline $\mathbf{C}$ & $\mathbf{M n}$ & $\mathbf{S i}$ & $\mathbf{S}$ & $\mathbf{P}$ & $\mathbf{C r}$ & $\mathbf{N i}$ & Mo & Co \\
\hline 0,12 & 0,9 & 0,36 & 0,007 & 0,017 & 9,33 & 0,49 & 0,51 & 0,86 \\
\hline
\end{tabular}

\begin{tabular}{l|l|l|l|l|l|l|l|l}
\hline $\mathbf{N b}$ & $\mathbf{C u}$ & $\mathbf{W}$ & $\mathbf{V}$ & $\mathbf{N}$ & $\mathbf{B}(\mathbf{p p m})$ & $\mathbf{A l}(\mathbf{p p m})$ & $\mathbf{T i}(\mathbf{p p m})$ & $\mathbf{M n + N i}$ \\
\hline 0,05 & 0,08 & 1,89 & 0,21 & 0,052 & 20 & 10 & 300 & 1,39 \\
\hline
\end{tabular}

La composición química posee la base de un metal de soldadura del tipo T/P92 comercial con el contenido de boro restringido a $20 \mathrm{ppm}$. Las diferencias se encuentran en el Ti, que ingresa a la composición como residual producto del rutilo (óxido de titanio) utilizado para la formación de escoria de protección de la pileta líquida durante el proceso de soldadura FCAW-G y el Co que se agrega para mejorar las propiedades de dureza e impacto a temperatura ambiente dado su control en la formación de la fase $\delta$-ferrita durante la solidificación [17]. En cuanto a los requisitos máximos del contenido de $\mathrm{Mn}+\mathrm{Ni}$ (1,5\% en peso) está relacionado con que las combinaciones de estos elementos tienden a bajar la temperatura AC1 aproximándola a la temperatura de PWHT y posibilitando con esto la formación de martensita fresca (PWHT sub-críticos - por debajo de AC1) [13].

\subsection{Microscopia electrónica de barrido (SEM) y análisis de difracción de electrones retrodifundidos (EBSD)}

En la micrografía de la Figura 4a, correspondiente al PWHT convencional, se observa una microestructura mixta de granos de martensita revenida columnares y equiaxiados y precipitados inter e intragranulares. En la muestra con el PWHT alternativo (Figura 4b) se aprecia granos de martensita revenida equiaxiados con precipitados inter e intragranulares. Este hecho estaría asociado al solubilizado realizado en el tratamiento alternativo, el cual tiende a eliminar los granos columnares.

Según se reporta [18-19], y cómo es posible apreciar en la figura 4, los precipitados localizados en lo que fueron los bordes de grano austenítico primario son de morfología elipsoidal $\left(\mathrm{M}_{23} \mathrm{C}_{6}\right)$ a diferencia de los intragranulares que también serían del mismo tipo, pero su morfología es circular. Estos precipitados presentan una composición compleja $(\mathrm{Fe}, \mathrm{Cr}, \mathrm{W}, \mathrm{Mo})_{23} \mathrm{C}_{6} \mathrm{y}$ tienden a crecer fácilmente con la temperatura de ensayo [20-22]. Los MX en cambio, presentan un tamaño mucho menor que $\operatorname{los} \mathrm{M}_{23} \mathrm{C}_{6}$, siendo difíciles de visualizar con microscopía SEM. Se componen de $(\mathrm{M}=\mathrm{V}, \mathrm{Nb}, \mathrm{Cr}$ y $\mathrm{X}=\mathrm{C}, \mathrm{N})$, se localizan principalmente en los bordes de los listones de martensita y su crecimiento es más lento [20-22]. 
Las dos muestran analizadas en este trabajo presentan similar densidad de partículas en segunda fase como se observa en las Figuras $4 \mathrm{a}$ y $4 \mathrm{~b}$ ampliadas.

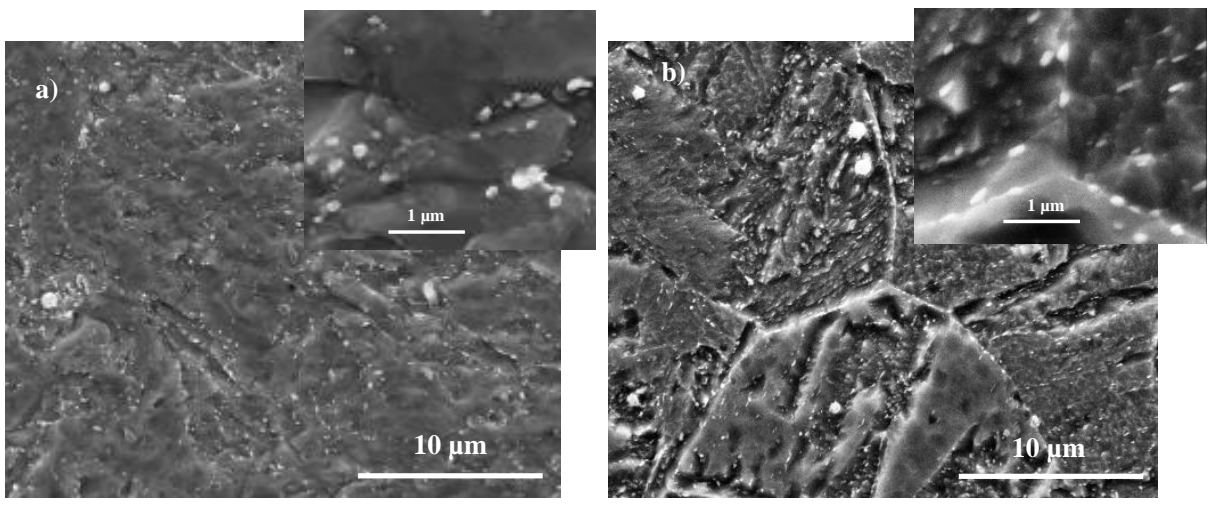

Figura 4: Micrografias SEM a) PWHT convencional; b) PWHT alternativo

La figura 5 presenta una reconstrucción cristalográfica de los granos austeníticos primarios a partir de mapas EBSD. La reconstrucción se basa en la relación de las orientaciones ideales entre ferrita y austenita como las descriptas por Kurdjumov-Sachs (KS) y Nishiwama-Wasserman (NW) [23]. En ellas se relacionan planos y direcciones de la ferrita que son paralelos a los de la austenita primaria y que son la base de la reconstrucción planteada a partir de la figura de polos EBSD. Según este análisis, se puede observar que la microestructura con PWHT alternativo (Figura 5b) muestra cualitativamente una distribución de tamaños más homogénea respecto de la muestra con PWHT convencional (Figura 5a) que denota una mayor dispersión. Este hecho podría haber provocado que los precipitados a su alrededor estuvieran más espaciados logrando disminuir con esto la velocidad de coalescencia de los mismos durante la degradación a alta temperatura para el PWHT alternativo.
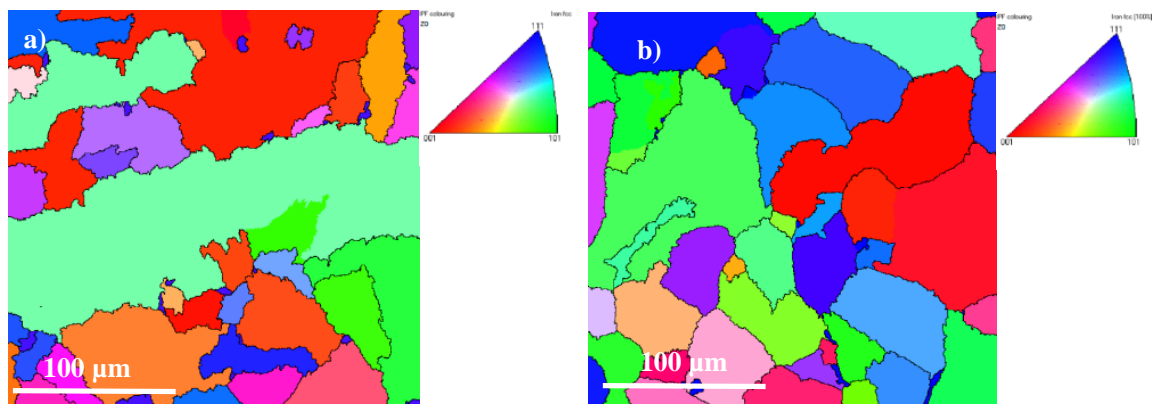

Figura 5: Reconstrucción cristalográfica de granos austeníticos primarios a partir de mapas EBSD a) PWHT convencional, b) PWHT alternativo.

En las Figuras 6a y 6c, se puede ver en los mapas de EBSD para el caso del PWHT convencional amplias regiones con una misma orientación cristalográfica y un marcado alineamiento de los dominios dentro de los contornos de los bordes de grano austenítico primario (dominio cristalino amplio con tendencia a un alineamiento). Este alineamiento no se observa en el PWHT alternativo (Figuras 7a y 7c). Esta cuestión podría reflejar una mejora de las propiedades de esta última condición, dado que se estaría impidiendo la formación de sistemas de deslizamiento de planos.

Desorientaciones de los planos cristalinos respecto de la orientación de referencia provocan discontinuidades en la red cristalina que se evidencian en bordes de diferente energía presentes en la microestructura [24]. Estos bordes se pueden clasificar según el ángulo girado de planos contiguos de una misma fase. Ángulos mayores a los $15^{\circ}$ representan bordes de alto ángulo [24] (HAGB - líneas negras en los mapas de bordes, Figuras 6 b y 7 b), en este caso atribuidos a bordes de grano austenítico primario y de placas de martensita, son de alta energía y nuclean por lo general el colapso de la estructura [4]. Ángulos mayores a $2^{\circ}$ y menores a $15^{\circ}$ 
se consideran de bajo ángulo o borde de subgrano [24] (LAGB- líneas claras en los mapas de bordes, Figuras 6 b y 7 b) son de baja energía y se los relaciona con la densidad de dislocaciones presentes en la microestructura [25]. Es bien conocido que las dislocaciones son sitios de nucleación de precipitados MX y que estos últimos impiden su movimiento durante la degradación [2-4].
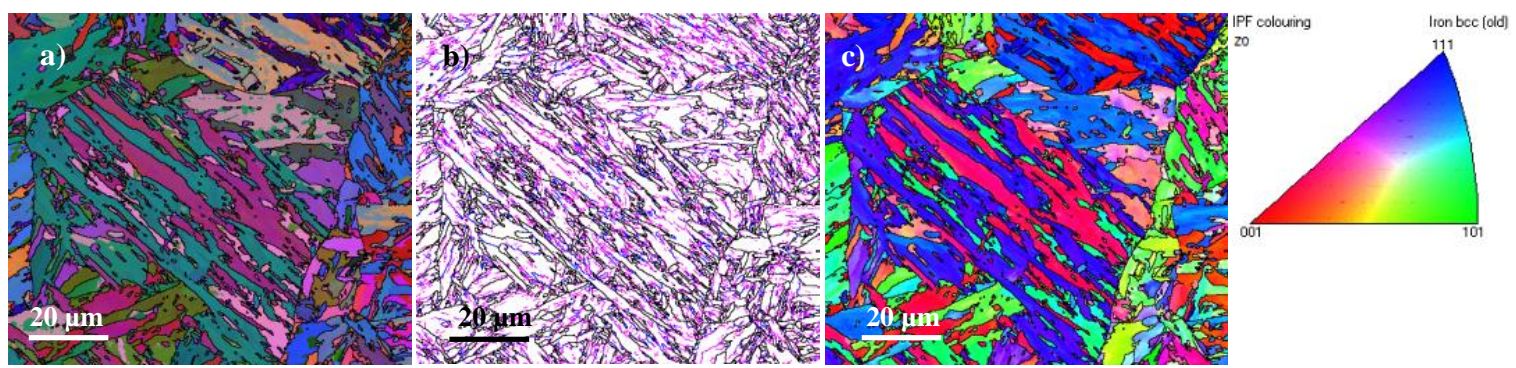

Figura 6: Mapas EBSD de la condición PWHT convencional, a) ángulos de Euler, b) bordes (líneas negras: alto ángulo, líneas azules: ángulo medio, líneas rosas: bajo ángulo), c) imagen de polos en dirección normal a la superficie (IPF).
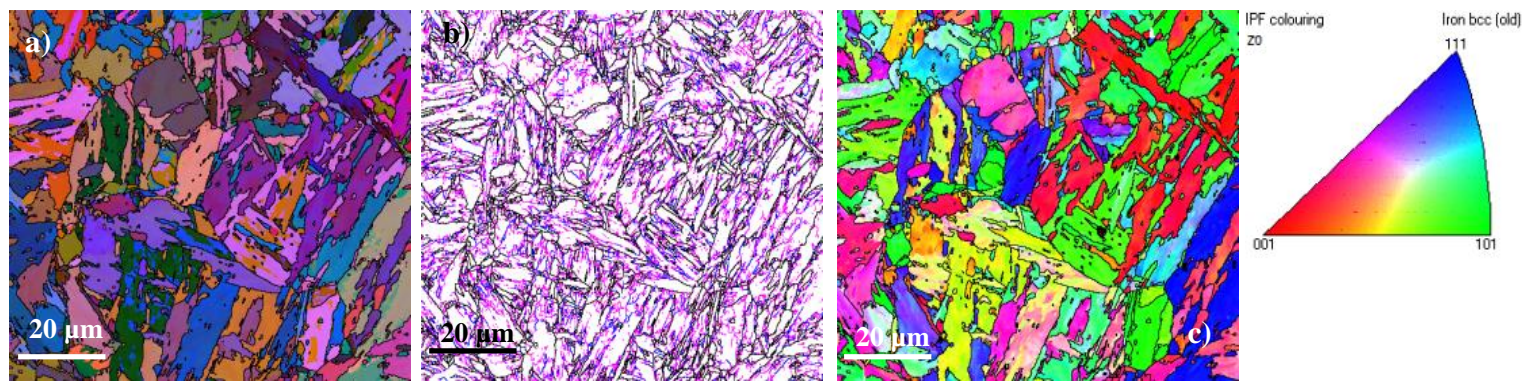

Figura 7: Mapas EBSD de la condición PWHT alternativo, a) ángulos de Euler, b) bordes (líneas negras: alto ángulo, líneas azules: ángulo medio, líneas rosas: bajo ángulo), c) imagen de polos en dirección normal a la superficie (IPF).

De acuerdo con estas apreciaciones y del análisis de los histogramas de dispersión de bordes (Figura 8) se puede ver que los bordes presentes en las dos muestras se concentran en los de bajo ángulo en torno a los $7^{\circ} \mathrm{y}$ los de alto ángulo en torno a los $58^{\circ}$. El PWHT alternativo generó en la microestructura una disminución de la frecuencia de bordes de alto ángulo y un aumento de los de bajo ángulo respecto del PWHT convencional. Esta observación se relacionaría directamente con la presencia de una mayor densidad de dislocaciones y una mayor probabilidad de dispersión de carburos finos del tipo MX en las muestras con un PWHT alternativo, lo que llevaría a alcanzar una mayor resistencia mecánica durante la degradación termomecánica de la microestructura asociada a esta condición [26-27]. 


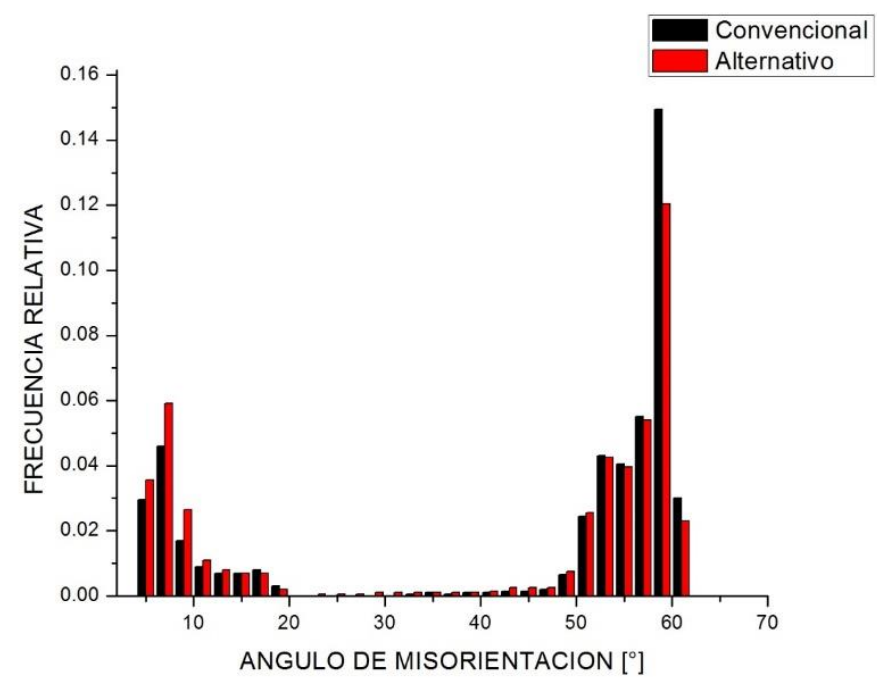

Figura 8: Histograma de ángulos de misorientación - comparación de los 2 diferentes PWHT (convencional - alternativo)

Si se consideran dos redes cristalinas de la misma fase que se encuentran superpuestas y se registra que existe un giro de una respecto de la otra manteniendo un átomo sin cambiar de sitio, se está en presencia de un borde especial denominado sitio de red coincidente (CSL) denotado con $\Sigma$. Dependiendo de los átomos que cambian de posición hasta que coincide el primero, se le asignan números $(1,3,5$, etc.) que reflejan de alguna manera el ángulo girado. Los CSL son límites de muy baja energía y provocan el bloqueo de las dislocaciones de forma más efectiva que un límite de grano convencional [25]. Se ha observado precipitación de segundas fases en límites con relación de coincidencia $\Sigma 3$ [25]. Para los casos aquí analizados se puede ver en la Figura 9 que existe una mayor densidad de estos bordes (predominio del tipo $\Sigma 3$ ) en la muestra con PWHT convencional. Sin embargo, se reporta que la combinación de estos bordes especiales en microestructuras con alta fracción de bordes de alto ángulo no genera un efecto beneficioso en relación a las propiedades a alta temperatura [25].
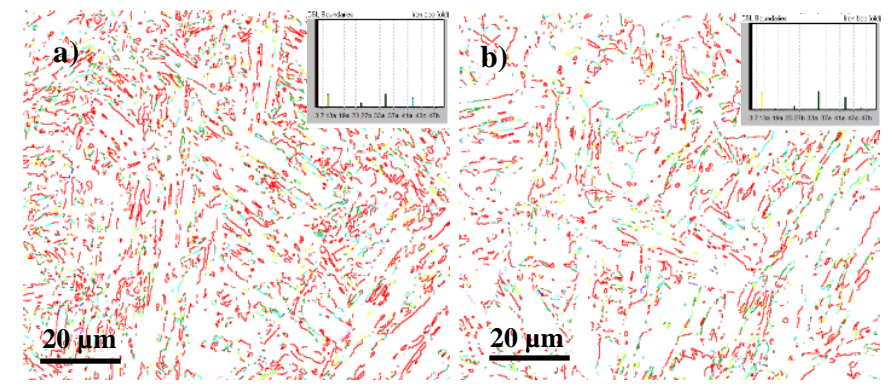

Figura 9: Mapas EBSD, bordes CSL (Sitios de Red Coincidentes). Líneas rojas CLS del tipo $\Sigma 3$ a) PWHT convencional b) PWHT alternativo

\subsection{Ensayos de tracción en caliente (HTT)}

En la Tabla 4 muestra los resultados (tensión máxima y tiempo a la tensión máxima) obtenidos de los ensayos de HTT para ambos PWHT y las distintas condiciones de ensayo (Temperatura y velocidad de deformación inicial).

Puede verse que la tensión máxima aumenta con la velocidad de deformación y disminuye con la tem- 
peratura. A su vez, el tratamiento alternativo mostró valores mayores que el convencional. En cuanto al tiempo a carga máxima el tratamiento alternativo mostró mayores valores que el convencional.

Con el objetivo de obtener una aproximación a parámetros de creep que caractericen el comportamiento de este material en servicio, se utilizó la teoría de correlación de HTT-Creep [8-12]. En ella se destaca que al igual que ocurre en la etapa secundaria de creep donde se registra una compensación de fenómenos de endurecimiento y recuperación que provocan una velocidad de deformación constante, en un ensayo de HTT se verifican fenómenos similares cuando se alcanza la tensión máxima en el material. En este punto la tensión, la velocidad de deformación y la temperatura son constantes (antes del comienzo de la estricción).

Las hipótesis sobre las que se basa este planteo son las siguientes:

1. La velocidad de deformación impuesta en el ensayo de HTT se correlaciona con la velocidad mínima de creep alcanzada en un ensayo de rotura al creep (CRT) a la misma temperatura y tensión aplicada.

2. La tensión máxima alcanzada en el ensayo de HTT se correlaciona con la tensión aplicada en un ensayo de CRT a la misma temperatura.

3. El tiempo en que se alcanza la tensión máxima en HTT se correlaciona con el tiempo necesario para provocar la rotura de la muestra en un ensayo de CRT a igual tensión y temperatura.

Los datos obtenidos de los ensayos de HTT fueron parametrizados según modelo de Larson-Miller para llevarlos a escenarios de creep empleando la Ecuación (1)

$$
P_{L M}=T \cdot(C+\log t) \cdot 10^{-3}
$$

donde $P_{L M}$ es el parámetro de Larson-Miller, $T$ es la temperatura $[\mathrm{K}], t$ es el tiempo [h] y $C$ es una constante característica del material que se obtiene a partir de curvas de isotensión en ensayos de creep [28].

Para la parametrización con el modelo de Larson-Miller se utilizó un valor de la constante $C$ mayormente aplicado en la bibliografía para metal de aporte puro de soldaduras T/P92, de similar composición a la aquí empleada [28]. Existe una dependencia lineal del valor $C$ con los tiempos de duración con que se quiere estimar la parametrización [28]. Se eligió para este caso un valor de $C=30$ normalmente aplicado para duraciones de 105 h. La Tabla 4 muestra los resultados para el parámetro de Larson-Miller obtenidos.

Tabla 4: Propiedades mecánicas a temperaturas elevadas de ambos PWHT (HTT y parametrización).

\begin{tabular}{|c|c|c|c|c|c|c|}
\hline \multirow[t]{2}{*}{ PWHT } & \multirow{2}{*}{\begin{tabular}{|l|} 
TEMPERATURA \\
[ $\left.{ }^{\circ} \mathbf{C}\right]$ \\
\end{tabular}} & \multirow{2}{*}{\begin{tabular}{|l|}
$\begin{array}{l}\text { VELOCIDAD DE } \\
\text { DESPLAZAMIEN- } \\
\text { TO }\end{array}$ \\
{$[\mathrm{mm} / \mathrm{s}]$}
\end{tabular}} & \multirow{2}{*}{\begin{tabular}{|l}
$\begin{array}{l}\text { VELOCIDAD DE } \\
\text { DEFORMACIÓN } \\
\text { INICIAL (६) }\end{array}$ \\
{$[1 / s]$}
\end{tabular}} & \multirow{2}{*}{\begin{tabular}{|l|}
$\begin{array}{l}\text { TENSIÓN } \\
\text { MAX. (Rm) }\end{array}$ \\
[MPa]
\end{tabular}} & \multirow{2}{*}{\begin{tabular}{|l} 
TIEMPO A \\
CARGA MÁ- \\
XIMA (T $\left.T_{\mathrm{Rm}}\right)$ \\
{$[\mathrm{s}]$}
\end{tabular}} & \multirow[t]{2}{*}{$\begin{array}{l}\text { PARÁMETRO DE } \\
\text { LARSON-MILLEF } \\
\text { (C=30) }\end{array}$} \\
\hline & & & & & & \\
\hline \multirow{9}{*}{ Convencional } & 625 & 5 & 1,39E-02 & 428 & 2,55 & 24,1 \\
\hline & 625 & 1 & $2,78 \mathrm{E}-03$ & 398 & 12,65 & 24,7 \\
\hline & 625 & 0,1 & $2,78 \mathrm{E}-04$ & 331 & 90,64 & 25,5 \\
\hline & 625 & 0,02 & $5,56 \mathrm{E}-05$ & 292 & 540,43 & 26,2 \\
\hline & 650 & 5 & 1,39E-02 & 377 & 2,70 & 24,8 \\
\hline & 650 & 5 & $1,39 \mathrm{E}-02$ & 379 & 3,55 & 24,9 \\
\hline & 650 & 1 & $2,78 \mathrm{E}-03$ & 346 & 12,21 & 25,4 \\
\hline & 650 & 0,1 & 2,78E-04 & 268 & 85,48 & 26,2 \\
\hline & 650 & 0,02 & $5,56 \mathrm{E}-05$ & 244 & 492,19 & 26,9 \\
\hline \multirow{2}{*}{ Alternativo } & 625 & 5 & $1,39 \mathrm{E}-02$ & 471 & 5,09 & 24,4 \\
\hline & 625 & 1 & 2,78E-03 & 502 & 13,69 & 24,8 \\
\hline
\end{tabular}




\begin{tabular}{l|l|l|l|l|l|l}
\hline PWHT & TEMPERATURA & $\begin{array}{l}\text { VELOCIDAD DE } \\
\text { DESPLAZAMIEN- } \\
\text { TO }\end{array}$ & $\begin{array}{l}\text { VELOCIDAD DE } \\
\text { DEFORMACION } \\
\text { INICIAL ( }()\end{array}$ & $\begin{array}{l}\text { TENSIÓN } \\
\text { MAX. (Rm) }\end{array}$ & $\begin{array}{l}\text { TIEMPO A } \\
\text { CARGA MÁ- } \\
\text { XIMA (T }\end{array}$ & $\begin{array}{l}\text { PARÁMETRO DE } \\
\text { LARSON-MILLER } \\
\text { (C=30) }\end{array}$ \\
\hline & 0,1 & $2,78 \mathrm{E}-04$ & 458 & 141,84 & 25,7 \\
\hline 625 & 0,02 & $5,56 \mathrm{E}-05$ & 416 & 687,74 & 26,3 \\
\hline 650 & 5 & $1,39 \mathrm{E}-02$ & 487 & 3,15 & 24,9 \\
\hline 650 & 1 & $2,78 \mathrm{E}-03$ & 443 & 13,05 & 25,4 \\
\hline 650 & 0,1 & $2,78 \mathrm{E}-04$ & 381 & 164,40 & 26,5 \\
\hline 650 & 0,1 & $2,78 \mathrm{E}-04$ & 406 & 110,10 & 26,3 \\
\hline 650 & 0,02 & $5,56 \mathrm{E}-05$ & 271 & 722,20 & 27,1 \\
\hline
\end{tabular}

A partir de ello, convirtiendo los valores de resistencia HTT $(\mathrm{Rm})$ en tensiones aplicadas en un ensayo de creep (CRT), se obtuvieron las curvas mostradas en la Figura 10, para ambos PWHT analizados.

Para el PWHT convencional se obtuvo una curva similar a la que existe en la literatura para ensayos de CRT de un metal de soldadura T/P92 [28], en donde para una tensión de aproximadamente 250 MPa se obtiene un $P_{L M}=27$ igual al aquí registrado, validando la aplicación de esta metodología para estos materiales. Considerando el $\mathrm{P}_{\mathrm{LM}}$ a temperatura constante un indicador de los tiempos de rotura, para una dada tensión, las muestras con PWHT alternativo alcanzaron mayores tiempos de duración para todo el rango analizado. Esto pone de manifiesto una mayor resistencia al creep para este PWHT.

Por lo expuesto, un PWHT alternativo mejoró la resistencia al creep del metal de soldadura, lo que se podría traducir en una disminución de la velocidad mínima de creep. Este PWHT generó una microestructura con mayor cantidad de bordes de bajo ángulo, cuestión que implicaría cualitativamente contar con una mayor densidad de dislocaciones.

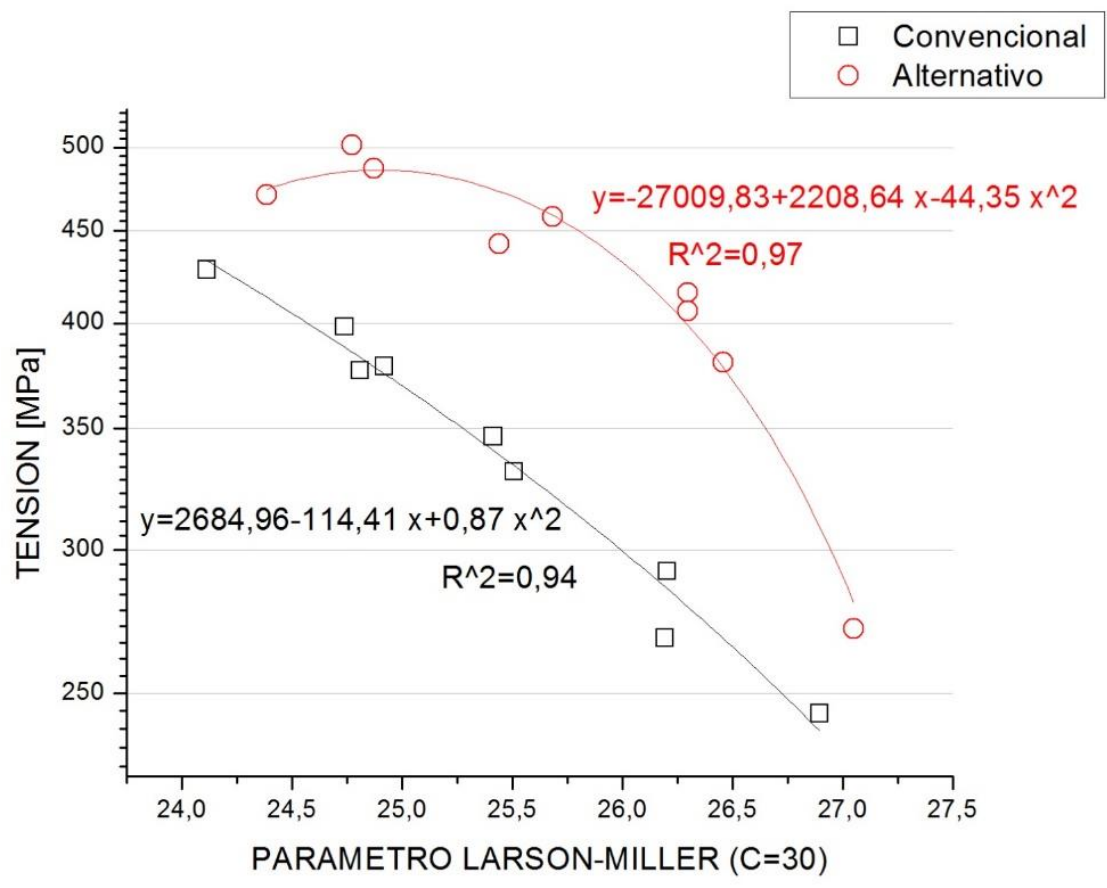

Figura 10: Parametrización con el modelo de Larson-Miller de los dos PWHT analizados

Esto último, en combinación con una mejor distribución de las partículas de segundas fases a lo largo de los bordes de granos austeníticos primarios y de placas de martensita habrían permitido un mejor funcio- 
namiento de los mecanismos de endurecimiento por dislocaciones y precipitados.

En contraposición el PWHT convencional generó una microestructura con mayor cantidad de bordes de alto ángulo que sumada a una orientación preferencial de los planos cristalinos posiblemente hayan propendido a la habilitación de sistemas de deslizamiento que limitaron el accionar de los mecanismos de endurecimiento, tal como se reflejó en sus propiedades mecánicas a altas temperaturas.

\section{CONCLUSIONES}

En este trabajo se encontró que la aplicación de un PWHT de solubilizado más un doble revenido, alternativo a los aplicados comúnmente a las soldaduras de aceros CSE9Cr, produce una mejora en las propiedades mecánicas a elevadas temperaturas.

El tratamiento de solubilizado generó en la microestructura una distribución de tamaños de granos austeníticos primarios más homogénea, lo que pudo haber provocado que los precipitados a su alrededor estuvieran más espaciados logrando disminuir con esto la velocidad de coalescencia de los mismos durante la degradación a alta temperatura.

La presencia de planos de una misma familia con cierto grado de alineamiento dentro de la microestructura podría estar provocando la generación de sistemas de deslizamientos que aceleraran el proceso de degradación mitigando la acción de los mecanismos de endurecimiento a alta temperatura. Esto se pudo observar en las muestras con PWHT convencional donde se registró una merma de propiedades en comparación al PWHT alternativo.

La microestructura a la que se le aplicó un PWHT alternativo, mostró un alto contenido de bordes de bajo ángulo producto de la misorientación de planos cristalinos contiguos, cuestión que pondría de manifiesto la presencia de una mayor densidad de dislocaciones, favoreciendo con esto uno de los mecanismos de endurecimiento del trabajo a altas temperaturas.

Un mayor contenido de bordes especiales del tipo $\Sigma 3$ como consecuencia de los CLS en combinación con un predominio de bordes de alto ángulo producto de la misorientación de planos cristalinos contiguos no aportó a la mejora en las propiedades, como se pudo comprobar en las muestras con PWHT convencional.

La utilización de ensayos de HTT junto a metodologías de parametrización de creep se muestra como una estrategia robusta para aproximar en tiempos cortos datos de fluencia lenta.

\section{AGRADECIMIENTOS}

Los autores agradecen a METRODE PRODUCTS LTD - UK, por la fabricación y provisión del consumible utilizado, a AIR LIQUIDE Argentina por la donación de los gases de soldadura, a CONARCO-ESAB por la realización de los análisis químicos, al INTI - MECÁNICA por la microscopía electrónica de barrido (SEM) y a los laboratorios de procesamiento y caracterización de metales del LABORATORIO NACIONAL DE NANOTECNOLOGÍA (LNnano) integrante del CENTRO NACIONAL DE INVESTIGACIÓN EN ENERGÍA Y MATERIALES (CNPEM) de Brasil por la realización de la microscopía SEM, análisis EBSD, la determinación de la composición química y los ensayos de tracción en caliente. Reconocen además a la ANPCyT por el soporte financiero, al programa BecAr 2013 "Estadías cortas en el exterior para doctorandos en ciencia, tecnología e innovación productiva" de la PRESIDENCIA DE LA NACIÓN ARGENTINA por la beca otorgada para la realización de los estudios en Brasil y a la UNIVERSIDAD NACIONAL DE LOMAS DE ZAMORA por el financiamiento de los mecanizados de las probetas.

\section{BIBLIOGRAFÍA}

[1] VISWANATHAN, R., HENRY, J.F., TANZOSH, J., et al., "U.S. program on materials technology for Ultra-Supercritical Coal Power Plants", Journal of materials engineering and performance, v. 14, n. 3, pp. 281-292, Jun. 2005.

[2] ABE, F., HORIUCHI, T., TANEIKE, M., et al., "Stabilization of martensitic microstructure in advanced 9Cr steel during creep at high temperature", Materials Science and Engineering, v. A378, pp. 299-303, Oct. 2004.

[3] HÄTTESTRAND, M., ANDRÉN, H.-O., "Boron distribution in 9 - 12\% chromium steels, Materials Science and Engineering, v. A270, pp. 33-37, Jun. 1999.

[4] HORIUCHI, M., IGARASHI, M., ABE, F., et al., "Improved utilization of added B in 9Cr heat-resistant steels containing W", ISIJ International, v. 42, Supplement, pp. S67-S71, 2002. 
[5] MARUYAMA, K., SAWADA, K., KOIKE, J-I., et al., "Strengthening Mechanisms of Creep Resistant Tempered Martensitic Steel”, ISIJ International, v. 41, n. 6, p 641-653, Feb. 2001.

[6] ABE, F., TABUCHI, M., TSUKAMOTO, S., et al., "Mechanisms for boron effect on microstructure and creep strength of ferritic power plant steels., Energy Materials, v. 4, n. 4, pp. 166-175, Ene. 2012.

[7] YIN, Y. F., FAULKNER, R.G., MORRIS, P.F., et al., "Modelling and experimental studies of alternative heat treatments in Steel 92 to optimize long term stress rupture properties, Energy Materials, v. 3, n. 4, pp. 232-242, Sep. 2008.

[8] BUENO, L. O., SOBRINHO, J.F.R., et al, "Correlation between creep and hot tensile behaviour for $2.25 \mathrm{Cr} 1 \mathrm{Mo}$ steel from $500^{\circ} \mathrm{C}$ to $700^{\circ} \mathrm{C}$ Part 1: An Assessment According to usual Relations Involving stress, temperature, strain rate and Rupture Time", Revista Matéria, v.7, n. 3, pp. 1098-1108, 2012.

[9] BUENO, L. O., SOBRINHO, J.F.R., et al., "Correlation Between Creep and Hot Tensile Behaviour for 2.25Cr-1Mo Steel from $500^{\circ} \mathrm{C}$ to $700^{\circ} \mathrm{C}$. Part 2: An Assessment According to Different Parameterization Methodologies", Revista Matéria, v.10, n. 3, pp. 463 - 471, 2005.

[10] BUENO, L. O., DIAS, C.R.F. "Equivalência entre dados de tração a quente e fluência para o cobre comercial puro. Parte 2: análise por diferentes metodologias de parametrização", Tecnol. Metal. Mater. Miner., v.8, n. 2, pp. 80-85, 2011.

[11] MORETO, J. A., CASTRO, D. B. V., BUENO, L. O., et al., "Correlação de dados de tração a quente e fluência para a liga Kanthal A1”, Metallurgy and Materials, v. 64, n. 2, pp. 181-186, 2011.

[12] SREENIVASAN, P. R. "Hot Tensile data and creep properties derived there form for $3161(\mathrm{~N})$ stainless steel with various nitrogen contents", Procedia Engineering, v. 55, pp. 82 - 87, 2013.

[13] ANSI/AWS A5.29/A5.29M:2010, "Specification for Low-Alloy Steel Electrodes for Flux Core Arc Welding", American Welding Society, 2010.

[14] ASTM A213 / A213M-15c, "Standard specification for seamless ferritic and austenitic alloy-steel boiler, superheater, and heat-exchanger tubes", ASTM International, West Conshohocken, PA, 2015.

[15] ASTM A335 / A335M-15a, "Standard specification for seamless ferritic alloy-steel pipe for hightemperature service", ASTM International, West Conshohocken, PA, 2015.

[16] ASTM E8 / E8M-15a, "Standard test methods for tension testing of metallic materials", ASTM International, West Conshohocken, PA, 2015.

[17] FAULKNER, J., WILLIAMS, J.A., GONZALEZ SANCHEZET, E., al. "Influence of Co, $\mathrm{Cu}$ and $\mathrm{W}$ on microstructure of 9\%Cr steel weld metals", Mate-rial Science and Technology, v. 19, pp. 347-354, 2003.

[18] VIJAYALAKSHMI, M., SAROJA, S., MYTHILI, R., et al. "Mechanisms and kinectics of tempering in weldments of 9 Cr-1Mo steel", J Nucl Mater, v. 279, pp. 293-300, 2000.

[19] ENNIS, P.J., "The creep rupture behaviour and steam oxidation resistance of P92 weldments". Materials at high temperatures, v. 23, n. 3-4, pp. 187-193, 2006.

[20] KANEKO K., MATSUMURA, S., SADAKATA, A., et al., "Characterization of carbides at different boundaries of 9Cr-steel", Materials Science \& Engineering, v. A374, pp. 82-89, 2004.

[21] TENEIKE M., ABE, F., SAWADA, K., et al., "Creep-strengthening of steel at high temperature using nano-size carbonitride dispersions", Nature, n. 424, pp. 294-296, 2003.

[22] YESCAS, M. A., MORRIS P. F., "Improved creep resistance of steel 92 by the use of modified heat treatments", ECCC Creep Conference, London, 2005.

[23] MAJID A., NELSON, T. W., SORENSEN, C.D., et al., "An approach to prior austenite reconstruction", Materials Characterization, v. 66, pp. 1-8, 2012.

[24] MALTLAND, T., SLTZMAN, S., "Electron Backscatter diffraction (EBSD) technique and materials characterization example", In: Zhou, W., Wang, Z. L. (eds), Scanning Microscopy for Nanotechnology: Techniques and Applications, chapter 2, New York, Springer, 2007.

[25] YARDLEY, V., SUGIURA, R., MATSUZAKI, T., et al., "Quantitative study of W-alloyed 9-12 Cr steel microstructures using EBSD”, Strength, Fracture and Complexity, v. 5, n. 1, pp. 39-52, 2007.

[26] TOKUNO K., HAMADA, K., UEMORI, R., et al., "A complex carbonitride of niobium and vanadium in 9\% Cr ferritic steels", Scripta Metallurgica et Materialia, v. 25, pp. 871-876, 1991.

[27] HAMADA K., TOKUNO, K., TOMITA, Y ., et al., "Effects of precipitate shape on high temperature strength of modified 9Cr-1Mo steels", ISIJ International, v. 35, n. 1, pp. 86-91, 1995. 
[28] ZHANG, Z. et al., "Properties of T/P92 steel weld metals for ultra supercritical (USC) power plant", Metrode Products Limited, UK. 